\title{
Integer Quadratic Programming (IQP) Model for Cut Order Plan
}

\author{
P.H.H.P.N. De Silva, G.H.J. Lanel and M.T.M. Perera \\ Department of Mathematics, University of Sri Jayewardenepura, Sri Lanka
}

\begin{abstract}
In the apparel industry Cut Order Planning (COP) considered as the most important procedure in the production process. Cutting large number of pieces with different shapes and sizes should be well planned to increase the utilization of fabric while reducing the wastage. Therefore cut planners pay great deal of attention to the number of plies, size ratios, length of the fabric, the cost of labour and they highly concerned about fabric wastage. This procedure is carried out manually in most of the factories. Due to that, high cost of labour, time and the fabric wastage have become more crucial problems in this industry. The existing manual procedure for COP was examined in this study and to overcome those issues an integer quadratic programming model was introduced for two and three dockets. As a result of this new model, user will receive number of plies that can be laid and size wise cutting templates that requires for the cutting process in order to minimize the cut panels wastage. Eventually this causes to increase efficiency and effectiveness of the cutting process more significantly. Keywords: Cut Order Plan, Dockets, Size Ratios
\end{abstract}

\section{Introduction}

Cut Order Plan takes a significant place in apparel industry. Low efficiency and high wastage of fabric consumption due to manual COP are critical problems in cutting process in this industry. In the cutting process if cut planners use less number of plies and excess number of templates it will be a wastage of labour and time otherwise using high number of plies one can obtain required number of cut panels without more labour and time. Deciding the number of plies and creating the cut order plan according to the size ratios which consistent with customer requirement, is depend on the planner's skill and experience due to unavailability of analytical method for COP. As a result, wastage of fabric and high cost of labour have become crucial issues in this industry. In this study minimizing the fabric cut panels and labour cost were consumed when developing a model for COP.

The variety of sizes, styles, fabrics, and colours induce significant complexity in this problem [1]. The number of blocks contain in the cut template from selected sizes is defined as the size ratio and size ratios are determined by the cut planners to develop the corresponding cut templates for a given cut order, as the major function [2].As discussed by Mausmi Ambastha, efficiency of COP depends on the number of plies and number of lays and also an inefficient COP courses substantial amount of fabric wastage [3]. According to the Fashion Capital web site "A docket is a form given to the manufacturer outlining the amount of garments required in different sizes and also the docket consists of fabric and trim samples as well as the production deadline and agreed price ensuring that customer and the manufacturer are working to the same information". In this research docket is defined as a document that contains full description of COP such as size ratios, number of plies, number of size wise cut templates, etc.

There are so many methods used to optimize the cut piece requirements. A Cut Order Plan is effective if it uses least number of plies and least number of lays while cutting an order [3]. Results revealed by the research [1], explored that heuristic methods are the only reasonable means of finding solutions for COP problems. According to the Kritsada Puasakul and Paveena Chaovalitwongse, most of the time COP is basically formulated as an integer nonlinear programming model (INLP) and required area of each part, demand quantity of part, maximum allowable area of each marker, maximum allowable ply height, minimum allowable ply height, and unit set up cost are used as the model parameters [4].In this research trial version of LINGO mathematical software is used to compute the results. LINGO is a commercial software that is highly supportive for optimization problems. The result window of this software provides detailed description of the results. It contains types of solution, objective value, objective bound, infeasibilities, extended solver steps, total solver iterations, elapsed run time in seconds, model class, description of variables and solutions. These details are highly supportive when comparing the efficiency and effectiveness of the manual and systematic COP.

\section{Methodology}

Data related to size wise order quantities were collected in retrospective manner. Ratio Creation in the COP depends on many factors such as size wise order quantities, usage of garments, maximum length of fabric, maximum number of panels, maximum number of plies and labour cost for time required to spread and cut fabrics. In this study, mainly considered the wastage of the fabric cut panels. An Integer Quadratic Programming 
(IQP) Model was developed having all the variables to decide the number of plies that should lay for the cut and number of cutting templates of each size.

Table 1: Cut ratio creation templates for two dockets

\begin{tabular}{|l|l|l|l|l|}
\hline & XS & S & M & L \\
\hline & $A$ & $B$ & $C$ & $D$ \\
\hline$x$ & $a$ & $b$ & $c$ & $d$ \\
\hline$y$ & $e$ & $f$ & $g$ & $h$ \\
\hline
\end{tabular}

$A, B, C, D$ are the size wise (XS-Extra Small, S-Small, M-Medium, L-Large) order quantities received from the customer.

$x, y$, denotes the number of plies and $a, b, c, d, e, f, g, h$, represent the number of size wise cutting templates respective to the plies.

Objective function was designed to minimize the fabric cut panels wastage $(w)$. Note that wastage could not be negative. The following is the objective function with respect to two dockets:

$w=x(a+b+c+d)+y(e+f+g+h)-(A+B+C+D):-(1)$

Number of plies can vary according to the cutting capacity and the fabric type. Therefore there should be a restriction for maximum number of plies $(p)$ and the minimum number of plies $(n)$

i.e.: $p \geq x_{s} y \geq n$ :- (2) Fabric length of lay for the cut must have a maximum value (q), so the quantities $k(a+b+c+d) \leq q:-(3)$ and $k(e+f+g+h) \leq q:-(4)$ where $\mathrm{k}$ constant which is usage of a garment.

According to the cutting department, cutting templates of the first docket should be greater than zero to fulfill the customer requirement. Usually shipments can't proceed without some number of all the sizes in the docket that customer required. Therefore $a_{x} b, c_{x} d \geq 1$ :- (5) and cutting templates in the second docket must be nonnegative i.e.: $\theta_{s} f, g, h \geq 0:-(6)$.

When creating the COP, planners pay attention to the first docket to achieve order quantity as much as possible and balance should be covered by the other dockets (in this model by second docket). Therefore constrain $x \geq y$ :- (7) is preferred in this model.

Since more plies are used for the first docket it gives,

$a+b+c+d \geq e+f+g+h:-(8)$

In order to increase efficiency and reduce labour cost, number of plies laid for the cut should be greater than or equal to the sum of size wise cut templates for each docket because fabric laying requires less time and labour, rather cutting fabric manually

i.e.: $x \geq a+b+c+d$ :- (9) and $y \geq e+f+g+h$ :- (10)

In this study fabric cut panels wastage was highly considered. Size wise wastage cannot be a negative value. It is preferable to have size wise wastage as zero, but if there is no optimal solution that gives wastage as zero, then the value of wastage should be greater than zero. Otherwise size wise order quantity cannot be achieved, therefore

$x_{\cdot} a+y_{\cdot} \cdot \bullet \geq A:-(11), x \cdot b+y \cdot f \geq B:-(12), x_{*} c+y \cdot g \geq C:-(13)$, and $x \cdot d+y \cdot h \geq D:-(14)$.

Finally IQP model for the two dockets is given below with inputs as maximum number of plies $(p)$ and minimum number of plies $(n)$, maximum length can lay $(q)$, usage of a garment $(k)$ and size wise order quantities $(A, B, C, D)$.

Objective function:

Minimize $w=x(a+b+c+d)+y(e+f+g+h)-(A+B+C+D)$

Subjects to:

$$
\begin{aligned}
& x \geq y \\
& x_{x} y \leq p \\
& x_{x} y \geq n \\
& a, b, c, d \geq 1 \\
& e_{x} f \cdot g, h \geq 0 \\
& k(a+b+c+d) \leq q \\
& k(e+f+g+h) \leq q \\
& x \cdot a+y \cdot e \geq A \\
& x \cdot b+y \cdot f \geq B \\
& x \cdot c+y \cdot g \geq C \\
& x \cdot d+y \cdot h \geq D \\
& x \geq a+b+c+d \\
& y \geq e+f+g+h \\
& a+b+c+d \geq e+f+g+h
\end{aligned}
$$


If the order can't be achieved by two dockets, then most of the time cut order plan can be achieved by three dockets.

Table2: Cut ratio creation templates for three dockets

\begin{tabular}{|l|l|l|l|l|}
\hline & XS & S & M & L \\
\hline & $A$ & $B$ & $C$ & $D$ \\
\hline$x$ & $a$ & $b$ & $c$ & $d$ \\
\hline$y$ & $e$ & $f$ & $g$ & $h$ \\
\hline$z$ & $i$ & $j$ & $l$ & $m$ \\
\hline
\end{tabular}

The following is the IQP model for three dockets.

Objective function:

Minimize $w=x(a+b+c+d)+y(e+f+g+h)+z(i+j+l+m)-(A+B+C+D)$

Subject to:

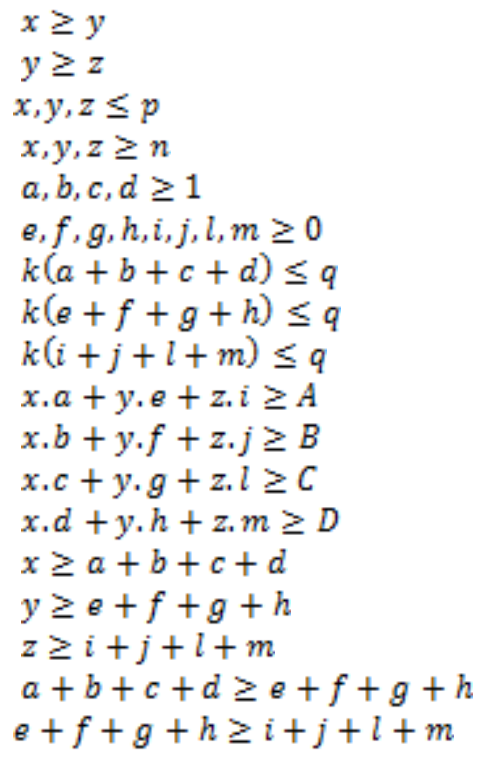

\section{Results And Discussion}

Secondary data was tested with the new model using LINGO mathematical software. The following are input data for a two dockets model and it is obtained by using collected ratio sheets of the company.

Usage of a garment $: 0.097 \mathrm{~m}$

Maximum length can lay $: 5 \mathrm{~m}$

Maximum number of plies : 50

Minimum number of plies : 5

Size wise order $\quad:$ XS $\quad$ S $\quad$ M $\quad$ L

$\begin{array}{llll}147 & 509 \quad 491 \quad 112\end{array}$

The following results were obtained from LINGO software

Objective function value

$: 0$

Table 3: Results of the model for two dockets

\begin{tabular}{|l|l|l|l|l|l|}
\hline $\begin{array}{l}\text { Docket } \\
\text { number }\end{array}$ & Number of plies & \multicolumn{4}{|c|}{ Cutting templates } \\
\hline $\mathbf{1}^{\text {st }}$ & $x$ & $a$ & $b$ & $c$ & $d$ \\
\cline { 2 - 6 } & 43 & 1 & 7 & 9 & 2 \\
\hline \multirow{2}{*}{$\mathbf{2}^{\text {nd }}$} & $y$ & $e$ & $f$ & $g$ & $h$ \\
\cline { 2 - 7 } & 26 & 4 & 8 & 4 & 1 \\
\hline
\end{tabular}

According to the results, it is clearly shows that this model provided a solution that does not waste any cut panels for this order.

The following table gives the manual output of COP for two dockets 
Table 4- Result of manual COP to the two dockets model

\begin{tabular}{|l|l|l|l|l|l|}
\hline $\begin{array}{l}\text { Docket } \\
\text { number }\end{array}$ & Number of plies & \multicolumn{4}{|c|}{ Cutting templates } \\
\hline \multirow{2}{*}{$\mathbf{1}^{\text {st }}$} & $x$ & $a$ & $b$ & $c$ & $d$ \\
\cline { 2 - 6 } & 30 & 4 & 14 & 14 & 1 \\
\hline \multirow{2}{*}{$\mathbf{2}^{\text {nd }}$} & $y$ & $e$ & $f$ & $g$ & $h$ \\
\cline { 2 - 6 } & 15 & 2 & 6 & 5 & 6 \\
\hline
\end{tabular}

When compare Table 3 and Table 4, the manual COP used total 45 plies with sixteen cut panels wastage and the new model provides total 69 plies without any fabric cut panels wastage. Even though the manual COP used less amount of plies it causes high fabric wastage. When compare the cutting templates new model provided only 36 templates whereas manual COP used 52. Consequently this results clearly state that manual cut order plan causes high fabric, time and labour wastage.

The following are input data for a three dockets model and it is obtained by using collected ratio sheets of the company.

Usage of a garment $: 1.00 \mathrm{~m}$

Maximum length can lay : $6 \mathrm{~m}$

Maximum number of plies : 60

Minimum number of plies : 5

$\begin{array}{llcll}\text { Size wise order } & : \text { XS } & \text { S } & \text { M } & \text { L } \\ & 123 & 190 & 189 & 207\end{array}$

$\begin{array}{llll}123 & 190 & 189 & 207\end{array}$

The following results were obtained from LINGO software

Objective function value: $\quad 0$

Table 5: Results of the model for three dockets

\begin{tabular}{|l|l|l|l|l|l|}
\hline $\begin{array}{l}\text { Docket } \\
\text { number }\end{array}$ & Number of plies & \multicolumn{4}{|c|}{ Cutting templates } \\
\hline $\mathbf{1}^{\text {st }}$ & $x$ & $a$ & $b$ & $c$ & $d$ \\
\cline { 2 - 6 } & 54 & 1 & 1 & 1 & 3 \\
\hline \multirow{2}{*}{$\mathbf{2}^{\text {nd }}$} & $y$ & $e$ & $f$ & $g$ & $h$ \\
\cline { 2 - 6 } & 45 & 0 & 2 & 3 & 1 \\
\hline \multirow{2}{*}{$\mathbf{3}^{\text {rd }}$} & $z$ & $i$ & $j$ & $l$ & $m$ \\
\cline { 2 - 6 } & 23 & 3 & 2 & 0 & 0 \\
\hline
\end{tabular}

As the result revealed by the model, it indicates that order quantity can't be achieved by two dockets due to unavailability of feasible solutions. Therefore the result is obtained using three dockets by completely eliminating the wastage.Consider the following table which gives manual output of COP in four dockets.

Table 6- Result of manual COP using four dockets instead of three dockets

\begin{tabular}{|l|l|l|l|l|l|}
\hline Docket number & Number of plies & \multicolumn{4}{|c|}{ Cutting templates } \\
\hline \multirow{2}{*}{$\mathbf{1}^{\text {st }}$} & $x_{1}$ & $a_{1}$ & $b_{1}$ & $c_{1}$ & $d_{1}$ \\
\cline { 2 - 6 } & 60 & 1 & 1 & 2 & 2 \\
\hline $2^{\text {nd }}$ & $x_{2}$ & $a_{2}$ & $b_{2}$ & $c_{2}$ & $d_{2}$ \\
\cline { 2 - 6 } & 35 & 1 & 1 & 2 & 2 \\
\hline \multirow{2}{*}{$\mathbf{3}^{\text {rd }}$} & $y$ & $e$ & $f$ & $g$ & $h$ \\
\cline { 2 - 6 } & 28 & 1 & 3 & 0 & 0 \\
\hline \multirow{2}{*}{$\mathbf{4}^{\text {th }}$} & $z$ & $i$ & $j$ & $l$ & $m$ \\
\cline { 2 - 6 } & 11 & & & 0 & 2 \\
\hline
\end{tabular}

This is a special situation. According to the cut planners skill and experience they used four dockets to achieve this order. When compare table 5 and 6, it is clearly shows that the same order can be achieved by three dockets as given by the new model. According to manual COP they used 134 plies and 19 cutting templates with wastage of six cut panels to fulfill the order. Nevertheless the results revealed by the new model shows that the order can be achieved by 122 plies and 17 cutting templates without any cut panels wastage. The manual COP result shows planner's skills and experience are not sufficient to get the maximum use of the fabric. Therefore our main objective of minimizing the fabric cut panels wastage is successfully fulfilled by these new models.

\section{Conclusion}

Creating an efficient and cost effective COP is a main role of cut planners in apparel industry. Cut planners should pay a great deal of attention to fabric utilization and cost of time and labour to spread and cut 
the fabric when creating the cut ratio. This research mainly focuses on reducing the wastage of cut panels. Using the proposed model with the support of LINGO mathematical software cut planners can plan the cut ratios more effectively and efficiently for two and three dockets with minimizing the fabric cut panels wastage.

\section{References}

[1]. J.C. Ammons and C. J. Blecha "Cut Order Planning-Short Term Task, Final Report" 1991.

[2]. R. P. Abeysooriya, T.G.I. Fernando "Canonical Genetic Algorithm To Optimize Cut Order Plan Solutions in Apparel Manufacturing", Journal of Emerging Trends in Computing and Information Sciences, vol. 3, no. $2,2012$.

[3]. M. Ambastha "Performance Measurement Tools-3 Cutting and Production Planning" 2012.

[4]. K. Puasakul and P. Chaovalitwongse - "The Review of Mark Planning Problem", Engineering Journal, vol. 20 issue 3, 2016.

[5]. W.K. Wong, C.K Chan, C.K. Kwong, P.Y. Mok, and W.H. Ip “ Optimization of manual fabric-cutting process in apparel manufacture using genetic algorithms" The International Journal of Advanced Manufacturing Technology, vol 27, issue 1, pp. 152$158,2005$.

[6]. G.H.J. Lanel, M.T.M. Perera “A Model to Optimize University Course Timetable Using Graph Coloring and Integer Linear Programming" IOSR Journal of Mathematics, vol 12, issue 5, pages 13-18, 2016. 